\title{
As dimensões raciais do processo de ratificação da Convenção do Genocídio nos Estados Unidos*
}

\section{The racial dimensions of the ratification process of the Genocide Convention in the United States}

\section{Resumo}

Ana Luiza Pinheiro Flauzina'

O presente artigo discute o longo processo de ratificação da Convenção para a Prevenção e a Repressão do Crime de Genocídio nos Estados Unidos, ressaltando os parâmetros racistas que o balizaram. Para tanto, explicitam-se as alegações de genocídio contra a população negra americana elaboradas dentro e fora do país e o impacto de tais denúncias nas discussões parlamentares e na interpretação judicial do genocídio.

Palavras-chave: Estados Unidos. Negros. Guerra fria. Convenção Para a Prevenção e Repressão do Crime de Genocídio.

\begin{abstract}
The article discusses the long process of ratification of the Convention the Prevention and Punishment of the Crime of Genocide in the United States high lighting its racist parameters. In order to do so, one explicits allegations of genocide against the American black population developed within and outside the country and the impact of such denunciations in parliamentary debates and judicial interpretation of genocide.
\end{abstract}

Keywords: United States. Blacks. Cold War. Convention on the Prevention and Punishment of the Crime of Genocide.

* Artigo recebido em fevereiro de 2013. Artigo aprovado em março de 2013.

1 Graduação em Direito pelo Centro Universitário de Brasília; graduação em História pela Universidade de Brasília; mestrado em Direito pela Universidade de Brasília; doutorado em Direito pela American University Washington College of Law; e pós-doutorado no African and African Diaspora Studies Department na University of Texas at Austin. 
Foi somente em fevereiro de 1986 que o Senado dos Estados Unidos ratificou a Convenção para a Prevenção e a Repressão do Crime de Genocídio. ${ }^{2} \mathrm{O}$ texto seria ratificado com reservas, sendo necessários ainda dois anos para que o Congresso finalmente aprovasse a legislação doméstica criminalizando o genocídio. ${ }^{3}$ Durante os longos debates nos quase quarenta anos que separaram a adoção da referida Convenção pelas Nações Unidas e sua ratificação pelos Estados Unidos, as preocupações em torno da interpretação do tipo penal e da extensão de seu reconhecimento estiveram sempre presentes.

Nesse processo, era claro o desconforto dos parlamentares com a possibilidade de indiciamentos de autoridades do governo e oficiais militares considerando a postura belicosa assumida pelo país durante a Guerra Fria e o delicado contexto racial que estruturava o cenário doméstico. Com relação à primeira questão, uma eventual caracterização das ações militares estadunidenses nas guerras da Coreia e do Vietnã como genocidas tornou-se dilema central, especialmente durante o período de 1950 até 1970. De fato, as preocupações dos congressistas provaram ser procedentes, considerando-se os intensos debates políticos em torno da aplicação da Convenção do Genocídio que marcaram o período em questão.

Um exemplo ilustrativo desse delicado ambiente político foi a criação do Tribunal Internacional de Crimes de Guerra do Vietnã também conhecido como Russell-Sartre Tribunal.Organizado por Bertrand Russell e Jean-Paul Sartre em novembro de 1966, o Tribunal consolidou-se como um fórum de debates público. Dezoito países foram representados por vinte e cinco indivíduos com reconhecido comprometimento com causas humanitá-

2 ORGANIZAÇÃO DAS NAÇÕES UNIDAS (ONU). Convenção para a prevenção e a repressão do crime de genocídio. Nova Iorque: ONU, 1948. Disponível em: <http://www.dhnet.org. br/direitos/sip/onu/genocidio/conv48.htm.> Acesso em: 20 fev. 2011.

3 CORNELL UNIVERSITY LAW SCHOOL. Legal Information Institute. 18 U.S.C. $\$ 1091$ - Genocide. Disponível em: <http://www.law.cornell.edu/uscode/text/18/1091> rias, reunindo-se em colegiado. ${ }^{4} \mathrm{Um}$ dos vereditos mais importantes dessa organização política foi a condenação unânime do governo dos Estados Unidos pela prática do crime de genocídio contra o povo do Vietnã. ${ }^{5}$

A famosa declaração de Jean-Paul Sartre, intitulada On Genocide, publicizada na segunda sessão do Tribunal em 1967, revela mais uma perspectiva em relação à interpretação da Convenção, dentre as muitas que marcam a história do genocídio como um fenômeno moderno. Para Sartre, os principais objetivos da guerra foram: "[...] a necessidade de estabelecer uma linha de defesa do Pacífico" ${ }^{6}$, tendo em vista o paradigma imperialista da Guerra Fria, aliado ao interesse econômico em se manter países subdesenvolvidos na órbita do capitalismo americano. ${ }^{7}$ Nesse contexto, a guerra do Vietnã cumpriu um papel simbólico, traduzindo-se como mensagem de retaliação dirigida às nações que estavam sob a zona de influência dos Estados Unidos.

A clara correspondência entre práticas militares empreendidas pelos Estados Unidos no Vietnã e os parâmetros estabelecidos pela Convenção do Genocídio foram destacadas pelo filósofo, que considerou que duas opções foram dadas à população vietnamita no Norte e no Sul do país: "morte coletiva ou desintegração". Portanto, em sua opinião, se Estados Unidos não era responsável por inventar o genocídio moderno, era defini-

4 Os participantes do comitê do Tribunal eram em sua maioria indivíduos politicamente identificados com organizações de esquerda. Muitos deles eram recipientes do Prêmio Nobel e outros prêmios internacionais humanitários. Não houve representantes dos vietnamitas ou nos Estados Unidos no painel. O Tribunal ouviu o testemunho de militares americanos, bem como das facções do Vietnã a fim de verificar as acusações de genocídio. Dentre os mais célebres representantes do comitê estavam: Simone de Beauvoir, Julio Cortázar, James Baldwin e Anders Gunther.

5 SARTRE, Jean-Paul. On genocide. Declaração feita na segunda sessão do Tribunal Internacional de Crimes de Guerra no Vietnã- Russell Bertrand, Dinamarca (Nov. 1967). Disponível em: <http://www.brusselstribunal.org/GenocideSartre.htm>.

6 SARTRE, Jean-Paul. On genocide. Declaração feita na segunda sessão do Tribunal Internacional de Crimes de Guerra no Vietnã- Russell Bertrand, Dinamarca (Nov. 1967). Disponível em: <http://www.brusselstribunal.org/GenocideSartre.htm>.

7 SARTRE, Jean-Paul. On genocide. Declaração feita na segunda sessão do Tribunal Internacional de Crimes de Guerra no Vietnã- Russell Bertrand, Dinamarca (Nov. 1967). Disponível em: <http://www.brusselstribunal.org/GenocideSartre.htm>.

8 SARTRE, Jean-Paul. On genocide. Declaração feita na segunda sessão do Tribunal Internacional de Crimes de Guerra no Vietnã- Russell Bertrand, Dinamarca (Nov. 1967). Disponível em: <http://www.brusselstribunal.org/GenocideSartre.htm>. 
tivamente "[...] culpado de ter optado por uma política de guerra e agressão que visa ao genocídio total a uma política de paz".

Embora recebendo pouca atenção nos Estados Unidos ${ }^{10}$, sendo qualificado como uma espécie de "show trial", o Russell-Sartre Tribunal teve significativo impacto internacional e é ilustrativo das disputas históricas em torno do conceito de genocídio. Cabe ainda sublinhar o especial significado da crítica às ações militares dos Estados Unidos ter tomado corpo na forma de um "julgamento", tendo em vista o fato da Guerra Fria ter praticamente paralisado o sistema jurídico internacional. ${ }^{11}$ Assim, além de importante declaração contra as violações de direitos humanos cometidas pelas forças militares estadunidenses no Vietnã, o Russell-Sartre Tribunal também pôs em cheque a postura omissa de instituições internacionais à época.

Se a preocupação com possíveis indiciamentos de autoridades governamentais resultantes das investidas militares no exterior agitavam os parlamentares, indubitavelmente, o principal obstáculo à ratificação da Convenção nos Estados Unidos girava em torno da possibilidade de sua aplicação tendo em vista as políticas raciais vigentes. ${ }^{12}$

As discussões travadas no Senado, especialmente nas primeiras décadas após a aprovação da Convenção pelas Nações Unidas, foram guiadas pelo temor de que

\footnotetext{
9 SARTRE, Jean-Paul. On genocide. Declaração feita na segunda sessão do Tribunal Internacional de Crimes de Guerra no Vietnã- Russell Bertrand, Dinamarca (Nov. 1967). Disponível em: <http://www.brusselstribunal.org/GenocideSartre.htm>.

${ }^{10}$ Mesmo que o Tribunal não tenha alcançado reconhecimento público nos Estados Unidos, não se deve subestimar seu impacto político. Como uma resposta direta ao tipo de interpretação do genocídio em tempos de guerra sustentada pelo Tribunal Russell-Sartre, o Congresso dos Estados Unidos formulou um entendimento quando da ratificação da Convenção das Nações Unidas nos seguintes termos: "(4) os atos cometidos no curso de conflitos armados praticados sem a intenção específica exigida pelo artigo II não são suficientes para constituir genocídio, conforme definido pela presente Convenção" Declarations and Reservations to the Convention on the Prevention and Punishment of the Crime of Genocide (Draft). prevent genocide int'l. Disponível em: <http://www. preventgenocide.org/law/convention/reservations/>.

${ }^{11}$ MAGOTO, Jackson. War crimes and realpolitik: international justice from World War I to the 21st century. Colorado: Lynne Rienner Publisher, 2004. p.127.

${ }^{12}$ CHURCHILL, Ward. A little matter of genocide: holocaust and the denial in the Americas 1942 to the present (2001). San Francisco: City Light Books, 2001. p.373-379.
}

os termos do instrumento legal incidissem sobre as políticas segregacionistas vigentes. ${ }^{13}$ Se a inquietude daquele contexto histórico trazia a questão para o debate público de maneira mais patente, é importante salientar que as conexões entre os ataques sistemáticos às comunidades negras nos Estados Unidos e a prática do que viria a ser definido como genocídio após a Segunda Guerra Mundial já vinham sendo desenhadas em décadas anteriores.

Nesse tocante, é oportuno se lembrar do acervo teórico e das práticas eugênicas que expandiam com vigor no inicio do século $\mathrm{XX}$, destacando a intensa comunicação entre eugenistas nos Estados Unidos e na Europa. Em resposta às críticas formuladas por eugenistas americanos, que desaprovavam o "excessivo" caráter antissemita do regime nazista, especialmente após a aprovação das Leis de Nuremberg em 1935, os propagandistas nazistas apontavam para as semelhanças entre as políticas adotadas contra a comunidade negra estadunidense e as empreendidas contra os judeus durante o Holocausto. ${ }^{14}$ De fato, após a década de 1930, a propaganda nazista tinha identificado os Estados Unidos como "[...] o principal ponto de referência, em razão de sua combinação específica de racismo étnico e eugênico". ${ }^{15} \mathrm{~A}$ imprensa alemã destacava a existência de linchamentos, restrições legais de casamento entre brancos e negros e a segregação como exemplos vivos da semelhança do programa racial que estava sendo levado a cabo contra os judeus no regime hitleriano.

Considerando as abundantes evidências de práticas genocidas cometidas em solo americano, os nazistas reagiram às críticas estadunidenses dirigidas ao seu programa racial destacando o duplo padrão que um autoproclamado Estado democrático assumia. Trata-se de uma lógica de crítica às políticas raciais adotadas no exterior e de contraditória manutenção do sistema racial doméstico. ${ }^{16}$

${ }^{13}$ CHURCHILL, Ward. A little matter of genocide: holocaust and the denial in the Americas 1942 to the present (2001). San Francisco: City Light Books, 2001. p.373-379.

${ }^{14} \mathrm{KUHL}$, Stefan. The nazi connection: eugenics, american racism, and german national socialism. Oxford: Oxford University Press, 1994. p.83.

${ }^{15}$ KUHL, Stefan. The nazi connection: eugenics, american racism, and german national socialism. Oxford: Oxford University Press, 1994p. 99.

${ }^{16} \mathrm{KUHL}$, Stefan. The nazi connection: eugenics, american racism, and german national socialism. Oxford: Oxford University Press, 1994 
Aqui, cabe pontuar que, nas primeiras décadas do século XX, o pensamento eugênico americano transformou-se em referência mundial, tendo um impacto direto na fundamentação do nazismo alemão. Como explica Edwin Black, “[...] a teoria, prática e legislação americana foram os modelos" ${ }^{17}$ em termos do pensamento eugênico que seria recepcionado em diferentes contextos sociais. ${ }^{18}$ No caso da Alemanha, os princípios eugênicos formulados nos Estados Unidos foram precedentes importantes para a configuração das políticas nazistas. ${ }^{19}$

O desenvolvimento desse sofisticado aporte teórico nos Estados Unidos foi em grande medida associado à existência de uma mentalidade eugênica traduzida em práticas de extermínio institucionais e privadas que tomaram um novo fôlego durante o período compreendido entre o fim da Guerra Civil até as primeiras décadas do século $\mathrm{XX} \cdot{ }^{20} \mathrm{~A}$ existência concomitante de práticas alicerçadas nos históricos padrões de desumanização dirigidos a corpos negros - dos espetáculos de linchamentos e execuções - aos procedimentos silenciosos nas prisões, e um conjunto de ideias eugênicas que situava a negros e negras na base da hierarquia racial não foi acidental.

Ao contrário, esse apetite pelo extermínio que impregnava o imaginário social fez com que o histórico terror de Estado dirigido às comunidades negras se estruturasse de maneira ainda mais incisiva. Nesse cenário,

${ }^{17}$ BLACK, Edwin. War against the weak: eugenics and America's campaign to create an master race. New York: Four Wall Eight Windows, 2003. p.258.

${ }^{18}$ A experiência brutal do nazismo criou a falsa percepção de que o movimento de eugenia, como um esforço intelectual e tecnológico, foi exclusivamente desenvolvido e vivenciado dentro das fronteiras europeias. O alcance global do movimento, que espalhou seus ideais desde o início do século XX como uma campanha formalizada e, a partir de meados do século XIX como uma inspiração e empreendimento teórico, foi traduzido em práticas políticas violentas que podem ser encontrados em países como Quênia, Argentina, França. Apesar da história desse audacioso empreendimento ter uma dimensão inegavelmente global, o envolvimento particular dos Estados Unidos em termos de conceituação, financiamento e experimentação de eugenia foi ímpar e esteve na base de sua estruturação. Ver: BLACK, Edwin. War against the weak. In: CAMPBELL, Chloe. Race and empire: eugenics in colonial Kenya. Manchester: Manchester University Press, 2007.

${ }^{19}$ BLACK, Edwin. War against the weak: eugenics and America's campaign to create an master race. New York: Four Wall Eight Windows, 2003. p.270.

${ }^{20}$ JAMES, Joy. Resisting state violence: radicalism, gender, and race in U.S. Culture. Minessota: University of Minessota Press, 1996. a tortura e a execução de corpos nas brutalidades do linchamento tornaram-se uma prática normalizada e racializada. ${ }^{21}$ Se durante o período da escravidão, a maioria das vítimas dos linchamentos eram brancos, depois da Guerra Civil, os assaltos passaram a ser direcionados a homens e mulheres negros, aumentando significativamente tanto em termos de número quanto em agressividade. ${ }^{22}$ Como Joy James salienta:

[...] no pós-guerra, quando a maioria das vítimas de linchamento era negra, a tortura, a mutilação, o desmembramento e a castração, assim como a decapagem das partes do corpo como lembranças, tornaram-se características regulares do linchamento. ${ }^{23}$

Nessa nova configuração, a precedência de práticas de extermínio sobre as características mais tradicionais de exploração das comunidades negras foi assumida por uma mentalidade eugenista que estava influenciando e servindo como justificativa das violações empreendidas. Nesse contexto, a implementação de políticas institucionais informadas por princípios eugênicos, tais como a adoção de leis de esterilização; ${ }^{24}$ as restrições no domínio

${ }^{21}$ JAMES, Joy. Resisting state violence: radicalism, gender, and race in U.S. Culture. Minessota: University of Minessota Press, 1996. p. 29.

${ }^{22}$ JAMES, Joy. Resisting state violence: radicalism, gender, and race in U.S. Culture. Minessota: University of Minessota Press, 1996

${ }^{23}$ JAMES, Joy. Resisting state violence: radicalism, gender, and race in U.S. Culture. Minessota: University of Minessota Press, 1996

${ }^{24}$ Ao todo, 23 estados adotaram leis de esterilização. Em 1925, 6.244 operações foram realizadas de acordo com a legislação eugênica. Em instituições mentais e asilos, o número de mortes de pacientes crescia exponencialmente. No Instituto de Illinois para os doentes mentais em Lincoln, Illinois, por exemplo, havia suspeita de que os pacientes foram deliberadamente expostos à tuberculose. De acordo com Edwin Black, “[...] na década de 1990, a expectativa média de vida para indivíduos com retardo mental era de 66,2 anos. Em 1930, a expectativa média de vida para aqueles classificados como débeis mentais era de cerca de 18,52 anos". BLACK, Edwin. War against the weak: eugenics and America's campaign to create an master race. New York: Four Wall Eight Windows, 2003. p. 256 
das políticas de imigração; ${ }^{25}$ e as regulamentações sobre casamentos inter-raciais, ${ }^{26}$ aumentaram sobremaneira a vulnerabilidade da população negra.

Esse atacado de políticas, compreendendo práticas de extermínio diretas e indiretas, seria exposto em nível doméstico e internacional. Se, antes da Segunda Guerra Mundial, o programa racial assumido no país não se destacava como exceção dada à reprodução comum de assaltos expressivos a populações negras em vários países europeus e, mais frequentemente, em seus domínios coloniais na África, o surgimento de uma ordem internacional baseada em princípios formais de igualdade expôs o sistema dos Estados Unidos como um paradoxo inevitável. Esse quadro mostrava-se particularmente perturbador na lógica da propaganda da Guerra Fria, visto que soviéticos e grupos pró-soviéticos em todo o mundo exploravam esse dado como uma demonstração das limitações das promessas capitalistas. ${ }^{27}$

Considerando esse clima hostil, era importante proteger as elites brancas de qualquer responsabilidade decorrente do sistema jurídico internacional. Essa pre-

\footnotetext{
${ }^{25}$ No domínio das políticas de imigração, a eugenia ajudou a moldar o tipo desejado de imigrante que seriam bem-vindos nos Estados Unidos, especialmente após a Primeira Guerra Mundial. A Lei de Imigração de 1924 reduziria radicalmente a imigração não nórdica, conectando-se com uma perspectiva que almejava transformar o perfil racial da sociedade americana de acordo com os parâmetros da supremacia branca. Esta visão foi sustentada por importantes autoridades norte-americanas, tais como Albert Johnson, um entusiasta eugenista, que se tornaria o presidente da Comissão de Imigração e Naturalização da Câmara dos Deputados. O deputado defendeu o carácter seletivo da Lei de Imigração de 1924 como uma necessária barricada contra "[...] um fluxo de sangue estrangeiro, com todos os seus equívocos herdados respeitando as relações de poder dos que governam para os governados". DANIELS, Roger. Guarding the golden door: american immigration policy and immigrants since 1882. Nova York: Hill and Wang, 2004. p.55; BLACK, Edwin. eugenics and America's campaign to create an master race. New York: Four Wall Eight Windows, 2003. p. 99, 123, 187-188.

${ }^{26}$ Para uma discussão sobre as regulamentações do casamento inter-racial nos níveis estadual e federal consular: GOLD, Susan. Loving V. Virginia: lifting the ban against interracial marriage. New York: Marshall Benchmark, 2008; VILLAZOR, Rose Cuison. The other loving: uncovering the federal government's racial regulation on marriage. New York University Law Review, v.86, p. 1361, 2011.

${ }^{27}$ MARTIN, Charles H. Internationalizing “The American Dilemma: the civil rights congress and the 1951 genocide petition to the United Nations. Journal of American Ethnic Hist., v. 16, n. 4, p. 35-54, jun. 1997. Disponível em: <http://www. accessmylibrary.com/article-1G1-20378734/internationalizing-american-dilemma-civil.html>
}

ocupação ficou ainda mais premente quando, além da exposição internacional, as práticas de terror impostas às comunidades negras no país também resultaram na apropriação do instrumento legal pela resistência negra doméstica que desafiava o status quo. Ativistas e intelectuais negros viram a Convenção como uma importante ferramenta para desafiar a realidade vivida pelas comunidades negras no país, formulando uma petição às Nações Unidas que impactaria significativamente o Governo americano.

Em 1951, sob a liderança de William Patterson, uma delegação do Civil Rights Congress (CRC) apresentou uma petição às Nações Unidas acusando o governo dos Estados Unidos da prática do crime de genocídio contra a população negra. Desde a formação da Organização das Nações Unidas, em 1945, organizações proeminentes começaram a chamar a atenção da comunidade internacional para as práticas de terror do Estado em curso nos Estados Unidos. Impulsionadas por WEB Du bois, The National Negro Congress (NNC) e a National Association for theAdvancementof Colored People (NAACP), também submeteram petições denunciando as políticas segregacionistas em 1946 e 1947 respectivamente..$^{28}$

O âmbito da petição de 1951, entretanto, foi o mais ambicioso, valendo-se da controversa Convenção do Genocídio e gerando intensas reanções nos planos interno e internacional. A petição, publicada por Patterson e ititulada We Charge Genocide: The Historic Petiton to the United Nations for Relieffrom a Crime ofthe United States Government Against the Negro People: ("Denunciamos o Genocídio: a Petição histórica para a Organização das Nações Unidas para o fim do Crime do Governo dos Estados Unidos contra o povo negro"), ${ }^{29}$ é considerada um marco na história do movimento dos direitos civis. ${ }^{30}$ Como uma denúncia histórica, a petição traz uma "perspectiva

\footnotetext{
${ }^{28}$ MARTIN, Charles H. Internationalizing “The American Dilemma: the civil rights congress and the 1951 genocide petition to the United Nations. Journal of American Ethnic Hist., v. 16, n. 4, p. 35-54, jun. 1997. Disponível em: <http://www. accessmylibrary.com/article-1G1-20378734/internationalizing-american-dilemma-civil.html>

${ }^{29}$ CIVIL RIGHTS CONGRESS. We charge genocide. New York: International Publishers, 1970.

${ }^{30}$ DAVIS, Angela. James and Esther Jackson: connecting the past to the present. American Communist History, v. 7, n. 2, p.271-273, 2008.
} 
multidimensional sobre o genocídio", ${ }^{31}$ apontando para a existência de práticas explícitas e tácitas de extermínio empreendidas pelo Estado com base nos fundamentos da desumanização negra. ${ }^{32} \mathrm{O}$ documento destaca os ataques institucionais às comunidades negras, incluindo a opressão econômica, a segregação residencial, os linchamentos e a privação dos direitos políticos, dentre outros. ${ }^{33}$ Além disso, a petição denuncia a prevalência de uma perspectiva fascista da política americana que resultava numa linha de continuidade de caráter racista: a perseguição de negros no plano doméstico e o ataque a populações não brancas no exterior. ${ }^{34}$

Embora o CRC não tenha sido capaz de apresentar a petição a qualquer agência da ONU, tal fato teve importantes consequências políticas como ressalta Charles Martin:

\begin{abstract}
Através de sua campanha contra o genocídio, o CRC brevemente expandiu as fronteiras intelectuais do debate contemporâneo racial, oferecendo o genocídio como uma teoria alternativa para explicar as relações raciais americanas. A aplicação deste novo conceito para a situação dos Afro-americanos, que ofendeu a muitos negros e brancos, adicionou uma perspectiva mais nítida, mais emocional ao crescente debate internacional sobre o racismo americano. Embora o estudo do CRC não tenha conseguido estabelecer que o governo dos Estados Unidos era culpado de cometer genocídio, o grupo identificou com precisão o governo federal como a entidade que possuía o poder suficiente para reduzir a enorme discriminação que, obviamente, existia. [...]. Por fim, a controvérsia sobre o genocídio definitivamente revelou que, até o final de 1951, a questão racial se tornou central na Guerra Fria. Desta feita, ajudando atransformar o "dilema americano" em um problema internacional, a Petição contra a prática do Genocídio demonstrou claramente que a discriminação racial em casa era o "calcanhar de Aquiles" da política externa americana. ${ }^{35}$
\end{abstract}

${ }^{31}$ VARGAS, Joao. Never meant to survive: genocide and utopias in black diaspora communities. Maryland. Rawman and Littlefield Publishers, 2008. p.4

32 VARGAS, Joao. Never meant to survive: genocide and utopias in black diaspora communities. Maryland. Rawman and Littlefield Publishers, 2008.

${ }^{33}$ CIVIL RIGHTS CONGRESS. We charge genocide. New York: International Publishers, 1970. p.1-19.

${ }^{34}$ CIVIL RIGHTS CONGRESS. We charge genocide. New York: International Publishers, 1970. p. 7.

${ }^{35}$ MARTIN, Charles H. Internationalizing "The American Dilemma": the civil rights congress and the 1951 genocide petition to the United Nations. Journal of American Ethnic Hist., v. 16, n. 4, p. 59, jun. 1997. Disponível em: <http://www.accessmylibrary.com/article-1G1-20378734/internationalizing-american-dilemma-civil.html>.
A inquietação promovida pela franca associação do genocídio com as políticas de extermínio dirigidas às comunidades negras fez mais do que simplesmente adiar a ratificação da Convenção pelos Estados Unidos. O temor de que as políticas segregacionistas pudessem ser analisadas nos termos da Convenção impactou a própria interpretação do instrumento legal e moldou as principais reservas e entendimentos que seriam formulados. ${ }^{36} \mathrm{Em}$ suma, a ratificação da Convenção sem quaisquer salvaguardas provou ser insustentável à luz do caráter explícito do genocídio imposto às comunidades negras no país.

Como forma de contornar as inconveniências jurídicas, foram criadas controvérsias em torno do artigo 2 ${ }^{\circ}$ da Convenção, inaugurando uma tendência que predomina nas interpretações hegemônicas sobre o genocídio na esfera judicial desde então. As discussões travadas no

${ }^{36}$ U.S. Declarations and Reservations to the Convention on the
Prevention and Punishment of the Crime of Genocide:
Reservations:
(1) That with reference to article IX of the Convention, before
any dispute to which the United States is a party may be sub-
mitted to the jurisdiction of the International Court of Justice
under this article, the specific consent of the United States is required in each case.

(2) That nothing in the Convention requires or authorizes legislation or other action by the United States of America prohibited by the Constitution of the United States as interpreted by the United States."

Understandings:

(1) That the term 'intent to destroy, in whole or in part, a national, ethnical, racial, or religious group as such' appearing in article II means the specific intent to destroy, in whole or in substantial part, a national, ethnical, racial or religious group as such by the acts specified in article II.

(2) That the term 'mental harm' in article II (b) means permanent impairment of mental faculties through drugs, torture or similar techniques.

(3) That the pledge to grant extradition in accordance with a state's laws and treaties in force found in article VII extends only to acts which are criminal under the laws of both the requesting and the requested state and nothing in article VI affects the right of any state to bring to trial before its own tribunals any of its nationals for acts committed outside a state. (4) That acts in the course of armed conflicts committed without the specific intent required by article II are not sufficient to constitute genocide as defined by this Convention.

(5) That with regard to the reference to an international penal tribunal in article VI of the Convention, the United States declares that it reserves the right to effect its participation in any such tribunal only by a treaty entered into specifically for that purpose with the advice and consent of the Senate.

Declarations and Reservations to the Convention on the Prevention and Punishment of the Crime of Genocide (Draft). Prevent Genocide Int'L.

Disponível em: <http://www.preventgenocide.org/law/ convention/reservations/>. 
Senado durante a década de 1950 revelam que os parlamentares debatiam para chegar a um acordo sobre uma interpretação aceitável dos elementos "intenção de destruir" e "em todo ou em parte" do artigo. ${ }^{37} \mathrm{Em}$ 1850, Dean Rusk, vice-subsecretário de Estado, prestou depoimento endossando a tradicional interpretação estrita de genocídio, afirmando que a intenção de destruir mencionada na Convenção estaria vinculada à destruição de todo o grupo. ${ }^{38}$ Esse entendimento teve influência considerável sobre um grande número de senadores que defenderam essa posição nas décadas seguintes. Embora alguns críticos, como representantes da American Bar Association, discordassem dessa perspectiva, salientando que o genocídio seria materializado com a intenção de destruir um grupo, num todo ou em parte, a questão do que deveria ser considerado como parte de um grupo persistia. ${ }^{39}$

Tendo em vista o caráter brutal dos ataques sobre grande parcela da população negra, a determinação de como se qualificar "uma parte de um grupo" para a caracterização do genocídio estava no centro das preocupações dos parlamentares. ${ }^{40}$ No entanto, a necessidade de minimizar a percepção global da resistência à ratificação da Convenção como uma associada ao temor de que oficiais americanos poderiam ser indiciados por essas práticas, fizeram com que o debate se estruturasse em torno da discussão do dolo, como Lawrence Le Blanc esclarece:

Talvez a razão para o atraso foi a de que as partes das audiências forneciam mais evidências do que as anteriormente publicadasde que uma importante base para a oposição à ratificação em 1950, era o medo de que a redação do Artigo II da Convenção fosse aplicável a linchamentos e motins raciais. O entendimento foi aparentemente proposto para amenizar tais temores. Mas a proposta da Comissão foi concebida de maneira ardilosa: para deixar claro que os Estados Unidos não interpretava a frase polêmica para que não se aplicasse a linchamentos e motins raciais, e afins, ou seja, para esclarecer o

${ }^{37}$ LEBLANC, J. Lawrence. The intent to destroy groups in the genocide convention: the proposed U.S. understanding. American Journal of International Law, n.78, p.369-370, apr. 1984.

${ }^{38}$ LEBLANC, J. Lawrence. The intent to destroy groups in the genocide convention: the proposed U.S. understanding. American Journal of International Law, n.78, p.373, apr. 1984.

${ }^{39}$ LEBLANC, J. Lawrence. The intent to destroy groups in the genocide convention: the proposed U.S. understanding. American Journal of International Law, n.78, p.313, apr. 1984.

${ }^{40}$ CHURCHILL, Ward. A little matter of genocide: holocaust and the denial in the Americas 1942 to the present (2001). San Francisco: City Light Books, 2001. p.374. significado de uma "parte" de um grupo, a comissão desenhou um projeto que modificou a noção da "intenção". ${ }^{41}$

Os Estados Unidos seriam, portanto, um precursor da atual tendência que marca a expansão da noção de crimes contra a humanidade e a manutenção de uma interpretação estrita do genocídio no Tribunal Penal Internacional. Aqui, é importante lembrar que o país desempenhou um papel importante na formulação de um “[...] princípio jurídico internacional através do qual a liderança nazista foi julgada em Nuremberg," ${ }^{42}$ defendendo uma interpretação ampla da então nebulosa categoria de crimes contra a humanidade. Além disso, os Estados Unidos litigou a necessidade de se formular legislação própria para a criminalização do genocídio, considerando a natureza excessivamente violenta do regime nazista. ${ }^{43}$

Assim sendo, durante os julgamentos de Nuremberg e outros que envolveram o destino de réus nazistas, como no caso de Adolf Eichmann ${ }^{44}$, o animus geral era fazer as interpretações jurídicas flexíveis a fim de acomodar a censura a violações de corpos europeus no contexto da II Guerra Mundial. No entanto, o novo status dos Estados Unidos no contexto da Guerra Fria exigiu uma mudança de postura em relação ao até então celebrado instrumento jurídico.

Nesse contexto, as reservas e entendimentos anexados à Convenção destinavam-se a autorizar ex-

${ }^{41}$ LEBLANC, J. Lawrence. The intent to destroy groups in the genocide convention: the proposed U.S. understanding. American Journal of International Law, n.78, p. 377, apr. 1984.

${ }^{42}$ CHURCHILL, Ward. A little matter of genocide: holocaust and the denial in the Americas 1942 to the present (2001). San Francisco: City Light Books, 2001. p. 364.

${ }^{43}$ CHURCHILL, Ward. A little matter of genocide: holocaust and the denial in the Americas 1942 to the present (2001). San Francisco: City Light Books, 2001.

${ }^{44}$ Uma boa ilustração da interpretação jurídica "flexível" utilizada na convicção de líderes nazistas é encontrada no julgamento de Adolf Eichmann. Após sua captura na Argentina, Eichmann foi levado para Israel a fim de enfrentar acusações criminais. A defesa alegou a ilegalidade de prisão e, principalmente, a questão da jurisdição para o julgamento do réu. Apesar da forte resistência à aceitação da jurisdição universal nos casos de genocídio, a Suprema Corte de Israel confirmou condenação de Eichmann, reconhecendo que os tribunais israelenses tinham o direito legal de exercer jurisdição com base em amplas disposições legais internacionais. Ver: SCHABAS, William. Judicial activism and the crime of genocide. In: DARCY, Shane; POWDERLY, Joseph. Judicial creativity at the international criminal tribunals. Oxford: Oxford University Press, 2010. p.63-70. 
plicitamente a violação indiscriminada de corpos, garantindo um passe livre para a edificação de máquinas renovadas de tortura em casa - mais bem simbolizada pelo complexo industrial prisional - e ao engajamento em investidas bélicas ilegais sob mutáveis rubricas que vão desde a derrota do comunismo, o tráfico de drogas e, mais recentemente, o terrorismo. Assim sendo, a insistência em parâmetros escritos para a configuração do dolo genocida está ligado ao desejo de garantir não apenas os padrões mais óbvios de impunidade, mas também de resguardar a conotação simbólica do delito num processo historicamente marcado pelas demandas da supremacia branca.

Considerando o espaço político ocupado pelos Estados Unidos durante a Guerra Fria, sua posição em relação à Convenção do Genocídio constituiria uma importante diretriz para as interpretações predominantes das disposições inscritas no instrumento jurídico. A esfera internacional rapidamente assimilaria o duplo padrão que afirma o confronto do racismo como uma premissa válida e, ao mesmo tempo, exclui o sofrimento negro de qualquer proteção jurídica eficaz. Uma lição aprendida em grande medida através do silenciamento das reivindicações formuladas pela resistência negra americana que denunciava a existência do genocídio.

Percebe-se, portanto, a conformação do referente legal de genocídio ao que Joy James chama de "semianalfabetismo" ${ }^{45}$ do discurso convencional em raça, em que a culminação do racismo antinegro em práticas genocidas é sistematicamente negada, especialmente quando perpetrado pelas elites brancas. ${ }^{46}$ Essa dinâmica revela que as restrições atuais impostas ao reconhecimento legal de genocídio como crime que vitimiza negros na diáspora não se baseia no alardeado refinamento jurisprudencial na matéria. Ao contrário, esse padrão segue um caminho histórico que, apesar das lutas de resistência, submeteu o controle sobre a vida das populações negras aos arranjos institucionais moldados pelo racismo. Nessa equação, a imunização do sistema jurídico internacional ao sofrimento negro tem desempenhado um papel eficaz

\footnotetext{
${ }^{45}$ JAMES, Joy. Resisting state violence: radicalism, gender, and race in U.S. culture. Minessota: University of Minessota Press, 1996. p.46.

${ }^{46}$ JAMES, Joy. Resisting state violence: radicalism, gender, and race in U.S. culture. Minessota: University of Minessota Press, 1996.
}

na atualização de relações de terror contra comunidades negras, especialmente no contexto da Diáspora.

\section{Referências}

BLACK, Edwin. War against the weak: eugenics and America's campaign to create an master race. New York: Four Wall Eight Windows, 2003.

CAMPBELL, Chloe. Race and empire: eugenics in colonial Kenya. Manchester: Manchester University Press, 2007.

CHURCHILL, Ward. A little matter of genocide: holocaust and the denial in the Americas 1942 to the present (2001). San Francisco: City Light Books, 2001.

CIVIL RIGHTS CONGRESS. We charge genocide. New York: International Publishers, 1970.

CONVENÇÃO para a prevenção e a repressão do crime de genocídio. Nova Iorque: ONU, 1948. Disponível em: <http://www.dhnet.org.br/direitos/sip/onu/genocidio/ conv48.htm.> Acesso em: 20 fev. 2011.

CORNELL UNIVERSITY LAW SCHOOL. Legal Information Institute. 18 U.S.C. \$ 1091 - Genocide. Disponível em: <http://www.law.cornell.edu/uscode/ text/18/1091>.

DANIELS, Roger. Guarding the golden door: american immigration policy and immigrants since 1882. Nova York: Hill and Wang, 2004.

DAVIS, Angela. James and Esther Jackson: connecting the past to the present, 7 Am. Communist Hist, n.. 2, 2008.

DECLARATIONS and Reservations to the Convention on the Prevention and Punishment of the Crime of Genocide (Draft). Prevent Genocide Int'l. $<$ http://www. preventgenocide.org/law/convention/reservations $>$.

GOLD, Susan. Loving V. Virginia: lifting the ban against interracial marriage. New York: Marshall Benchmark, 2008.

JAMES, Joy. Resisting state violence: radicalism, gender, and race in U.S. culture. Minessota: University of Minessota Press, 1996.

KUHL, Stefan. The nazi connection: eugenics, american racism, and german national socialism. Oxford: Oxford University Press, 1994.

LEBLANC, J. Lawrence. The intent to destroy groups in the genocide convention: the proposed U.S. understanding. American Journal of International Law, n.78. p. 369, apr. 1984 . 
MAGOTO, Jackson. War crimes and realpolitik: international justice from world war I to the $21^{\text {st }}$ century. Colorado: Lynne Rienner Publisher, 2004.

MARTIN, Charles H. Internationalizing "The American Dilemma": the civil rights congress and the 1951 genocide petition to the United Nations. Journal of American Ethnic Hist., v. 16, n. 4, jun. 1997. Disponível em: <http:// www.accessmylibrary.com/article-1G1-20378734/ internationalizing-american-dilemma-civil.html>

SARTRE, Jean-Paul. On genocide. Declaração feita na segunda sessão do Tribunal Internacional de Crimes de Guerra no Vietnã- Russell Bertrand, Dinamarca (Nov. 1967), Disponível em: <http://www.brusselstribunal.org/ GenocideSartre.htm>
SCHABAS, William. Judicial activism and the crime of genocide. In: DARCY, Shane; POWDERLY, Joseph. Judicial Creativity at the International Criminal Tribunals. Oxford: Oxford University Press, 2010.

VARGAS, Joao. Never meant to survive: genocide and utopias in black diaspora communities. Maryland: Rawman and Littlefield Publishers, 2008.

VILLAZOR, Rose Cuison. The other loving: uncovering the federal government's racial regulation on marriage. New York University Law Review, v. 86, p. 1361. nov. 2011. 


\section{Para publicar na revista Universitas/JUS, acesse $o$ endereço eletrônico www.publicacoesacademicas.uniceub.br.}

Observe as normas de publicação, para facilitar e agilizar o trabalho de edição. 